\title{
Thioredoxin 1 is associated with the proliferation and apoptosis of rheumatoid arthritis fibroblast-like synoviocytes
}

\author{
Tianbao Lu ${ }^{1} \cdot$ Ming Zong ${ }^{1} \cdot$ Shasha Fan ${ }^{1} \cdot$ Ying Lu $^{1} \cdot$ Shanhan Yu ${ }^{1} \cdot$ Lieying Fan ${ }^{1}$
}

Received: 30 March 2017 /Revised: 23 August 2017 / Accepted: 7 September 2017 /Published online: 15 September 2017

(C) The Author(s) 2017. This article is an open access publication

\begin{abstract}
We aimed to investigate the possible effects of thioredoxin $1(\operatorname{Trx} 1)$ on the proliferation and apoptosis of rheumatoid arthritis fibroblast-like synoviocytes (RA-FLSs) and elucidate the possible mechanisms involved. We investigated the distribution and expression of Trx 1 in synovial tissues from RA and osteoarthritis (OA) patients by immunohistochemistry and real-time polymerase chain reaction (RTPCR) analyses. RA-FLSs were isolated and cultured under normoxic ( $21 \%$ oxygen) or hypoxic (3\% oxygen) concentrations. Transfection of Trx1-siRNAs and a Trx1 overexpression construct was conducted to manipulate the expression of Trx1. Protein expression was detected by Western blot. Doxorubicin (Adriamycin, ADR) was used to induce apoptosis. LY-294002 was used for the inhibition of PI3KAkt. Cell proliferation and apoptosis were determined by MTS (3-[4,5-dimethylthiazol-2-yl]-5-[3carboxymethoxyphenyl]-2-[4-sulfophenyl]-2H-tetrazolium, inner salt) assay and flow cytometry, respectively. The mRNA and protein expression of Trx 1 in RA tissues was higher than that in OA tissues. The expression levels of Trx1 and cell proliferation in RA-FLSs were increased under hypoxia in comparison to those under normoxia. In hypoxia, downregulation of Trx 1 significantly suppressed FLS proliferation, and the expression of PI3Kp85, phospho-Akt, and Bcl-2, while notably increased FLS apoptosis and the expression of active Caspase 3 and Bax. In normoxia, Trx 1 overexpression
\end{abstract}

Lieying Fan

flieying@yeah.net

1 Department of Clinical Laboratory, Shanghai East Hospital, Tongji University School of Medicine, No. 150, Jimo Road,

Shanghai 200120, People's Republic of China promoted the FLS proliferation and the expression of PI3Kp85, phospho-Akt, and Bcl-2, but inhibited FLS apoptosis and the expression of active Caspase 3 and Bax in FLSs. Such effects were partially repressed by LY-294002 treatment. Trx 1 may play an important role in regulating the proliferation and apoptosis of RA-FLSs by modulating PI3K-Akt activation.

Keywords Apoptosis · Fibroblast-like synoviocytes · Proliferation $\cdot$ Rheumatoid arthritis $\cdot$ Thioredoxin 1

\section{Introduction}

Rheumatoid arthritis (RA) is a chronic inflammatory disorder and characterized by chronic joint inflammation and synovial hyperplasia, which ultimately leads to progressive destruction of articular cartilage and bone. The synovial hyperplasia is featured by an overabundance of RA fibroblast-like synoviocytes (RA-FLSs). RA-FLSs not only display tumor-like destructive and invasive features but also affect the inflammatory microenvironment through secreting a variety of proinflammatory factors or interacting with immune cells $[1,2]$. Therefore, RA-FLSs play an important role in the initiation and development of RA, and the therapy targeting RA-FLSs might improve clinical symptoms of inflammatory arthritis without inhibiting systemic immune responses [3].

Due to the enhanced proliferation of RA-FLSs, the oxygen consumption in RA synovium increases, which leads to synovial hypoxia and hypoperfusion [4]. In the 1970s, LundOlesen $\mathrm{K}$ et al. first revealed the hypoxic nature of RA synovium by measuring the oxygen tension in the synovial fluid of RA patients with a Clark-type electrode [5]. 
Subsequent studies have shown that synovial tissues in RA patients are hypoxic, with the oxygen tension of $2-4 \%$, as compared with that of $9-12 \%$ in patients without RA [6-9]. The hypoxic condition observed in RA synovium is likely to activate a transcriptional response mainly driven by hypoxiainducible factors (HIFs). Several signaling pathways responsible for RA-FLS proliferation have been proposed [2, 10], while the signaling pathways involved in RA-FLS proliferation under hypoxia are poorly understood. Hypoxia activates phosphatidylinositol 3-kinase (PI3K)-serine/threonine kinase (Akt) pathway $[11,12]$, which plays a pivotal role in cell proliferation, migration, invasion, and survival [13]. Recently, a study has reported that hypoxia-induced proliferation and invasion of RA-FLSs are mediated through the PI3K-Akt pathway, suggesting a critical role of this pathway in RA progression $[12,14]$.

Thioredoxin 1 (Trx1), a $12 \mathrm{kDa}$ protein with redoxactive dithiol in the highly conserved active site (CysGly-Pro-Cys), is ubiquitously expressed in all tissues of the human body [15]. Trx 1 is involved in redoxdependent signaling via regulating HIFs $[16,17]$ and PI3K-Akt pathway [18-20]. Trx1 levels have been found elevated in the synovial fluid, serum, and synovial tissues of RA patients [21-23]. Trx1 is known to be induced by hypoxia and by proinflammatory stimuli, both of which are features of RA synovia [22-24]. The roles of Trx1 overexpression in tumorigenesis have been widely investigated [24], while little is known about its function on hypoxiainduced RA-FLS proliferation. Our previous study based on proteomic analysis has found that the expression of Trx1 in RA-FLSs was higher than that in osteoarthritis (OA)-FLSs. The present study tested the hypothesis that Trx1 regulates the proliferation and apoptosis of RA-FLSs via PI3K-Akt pathway under normoxic ( $21 \%$ oxygen) or hypoxic (3\% oxygen) conditions.

\section{Materials and methods}

\section{Patients and controls}

Synovial tissues were obtained from six patients with RA (two males, four females, mean age: $65 \pm 9$ years) and six patients with osteoarthritis (OA) (three males, three females, mean age: $62 \pm 11$ years) who underwent knee arthroscopic or replacement surgery at Shanghai East Hospital. The clinical characteristics of these patients were listed in Table 1. All patients fulfilled the diagnosis of the American College of Rheumatology for RA [25] and OA [26]. Informed consent was obtained from each of the enrolled patients, and the study protocol was approved by the Ethics Committee of Shanghai East Hospital.

\section{Immunohistochemistry for synovial tissue}

All specimens were fixed in $10 \%$ neutral buffered formalin, embedded in paraffin, and cut into $5-\mu \mathrm{m}$-thick tissue sections. The tissue sections were deparaffinized and rehydrated for immunohistochemical staining. The sections were heated at $95{ }^{\circ} \mathrm{C}$ for 20 min with Dako Target Retrieval Solution (Dako, Copenhagen, Denmark). After blocking with 3\% $\mathrm{H}_{2} \mathrm{O}_{2}$, the sections were incubated overnight with anti-Trx1 (1:200, no . 2285, Cell Signal Technology Inc., USA) at $4{ }^{\circ} \mathrm{C}$. Following three rinses with phosphate-buffered saline (PBS), $15 \mathrm{~min}$ for each, the sections were incubated with secondary antibody (Envision ${ }^{\mathrm{TM}}$ Detection Kit, Dako) for $30 \mathrm{~min}$ at room temperature. Finally, after another three rinses with PBS, the sections were visualized by using diaminobenzidine substrate kit (Dako) according to the manufacturer's instructions.

\section{Quantitative RT-PCR analysis}

Total RNA was extracted from synovial tissues and FLSs using TRIzol ${ }^{\mathrm{TM}}$ (Invitrogen, Carlsbad, CA, USA), and reverse transcription was performed using first-strand cDNA Synthesis Kit (Takara, Dalian, China) according to the manufacturer's instructions. Real-time polymerase chain reaction (RT-PCR) was performed using Premix Ex Taq SYBR Green PCR (Takara) on an ABI PRISM 7500 (Applied Biosystems, Foster City, CA, USA) according to the manufacturer's instructions. The sequences of primers were used as follows: Trx1, forward 5'-AAGCCTTGGACGCTGCAG-3', reverse 5'-CATCCTGACAGTCATCCACATCTACT-3'; GAPDH, forward 5'-TGACTTCAACAGCGACACCCA-3', reverse 5'-CACCCTGTTGCTGTAGCCAAA-3'. GAPDH served as the internal control.

\section{Isolation and culture of RA-FLSs}

Synovial tissues were immediately placed in Roswell Park Memorial Institute (RPMI) 1640 medium (Life Technologies, Carlsbad, CA, USA) and processed within $4 \mathrm{~h}$. The tissues were minced and evenly spread on the bottom of cell culture flasks in RPMI 1640 medium at $37^{\circ} \mathrm{C}$ for $6 \mathrm{~h}$. Next, the tissues were incubated with RPMI 1640 medium supplemented with $10 \%$ fetal bovine serum and antibioticantimycotic solution (Invitrogen) at $37{ }^{\circ} \mathrm{C}$ in a humidified $5 \% \mathrm{CO}_{2}$ atmosphere. Non-adherent tissue pieces were carefully removed by replacing the medium every 3 to 5 days and passaged when the primary FLSs reached $70-80 \%$ confluence. FLSs were grown further over four to eight passages. To characterize the cytological phenotype of synovial cultures, the third passage cells were stained with mouse monoclonal antibodies $(\mathrm{mAb})$ against human $\mathrm{CD} 14$ and CD90 (9011-0149-025 and eBio5E10, respectively, eBioscience, San Diego, CA, USA) and the cells showed CD14 negative 
Table 1 Clinical and serological characteristics of the patients

\begin{tabular}{|c|c|c|c|c|c|c|c|c|c|}
\hline Patient no. & Diagnosis & Gender & Age (years) & $\begin{array}{l}\text { Disease duration } \\
\text { (year) }\end{array}$ & Medications taken & $\begin{array}{l}\mathrm{ESR} \\
(\mathrm{mm} / \mathrm{h})\end{array}$ & $\begin{array}{l}\mathrm{RF} \\
(\mathrm{IU} / \mathrm{mL})\end{array}$ & $\begin{array}{l}\text { CRP } \\
(\mathrm{mg} / \mathrm{L})\end{array}$ & $\begin{array}{l}\text { Anti-CCP } \\
\text { (IU/L) }\end{array}$ \\
\hline 1 & RA & Female & 72 & 15 & MTX + Leflunomide & 24 & 48.0 & 62.4 & 26.71 \\
\hline 2 & RA & Male & 48 & 8 & $\mathrm{MTX}+\mathrm{SSZ}$ & 36 & 127.8 & 33.4 & 38.3 \\
\hline 3 & RA & Female & 56 & 5 & SSZ + Leflunomide & 41 & 38.6 & 29.7 & 12.9 \\
\hline 4 & RA & Female & 69 & 12 & $\mathrm{MTX}+\mathrm{SSZ}$ & 29 & 71.6 & 72.5 & 35.8 \\
\hline 5 & RA & Female & 59 & 6 & MTX + Leflunomide & 19 & 68.4 & 33.8 & 14.6 \\
\hline 6 & RA & Male & 67 & 7 & MTX + Leflunomide & 51 & 112.2 & 48.6 & 35.8 \\
\hline
\end{tabular}

$R A$ rheumatoid arthritis, $M T X$ methotrexate, $S S Z$ sulfasalazine, $E S R$ erythrocyte sedimentation rate, $R F$ rheumatoid factor, $C R P$ C-reactive protein, anti$C C P$ anti-cyclic citrullinated peptide antibody

and CD90 positive as measured by flow cytometry (Beckman Coulter, Fullerton, CA, USA).

\section{Hypoxic conditions}

To imitate the joint cavity hypoxic microenvironment of RA patients, hypoxia $\left(3 \% \mathrm{O}_{2}\right)$ was induced by culturing cells inside a tri-gas incubator (Forma Scientific, Div. of Mallinckrodt, Inc., Marietta, Ohio) infused with a mixture of $3 \% \mathrm{O}_{2}, 5 \% \mathrm{CO}_{2}$, and $92 \% \mathrm{~N}_{2}$ at $37{ }^{\circ} \mathrm{C}$. The cells were cultured in a normal culture gas-mixture condition with $21 \% \mathrm{O}_{2}$ component were served as control.

\section{Transfection of siRNA and plasmid}

Predesigned specific small interfering RNA (siRNA) targeting human Trx1 mRNA (RefSeq NM 003329.3) (si-Trx1-1 and si-Trx1-2) and negative control siRNA (named NC) were synthesized by Genepharma Inc. (Shanghai, China). The targeting sequences as follows: si-Trx 1-1, 5'GUCAAAUGCAUGCCAACAUTT-3'; si-Trx1-2, 5'-CCAC CAUUAAUGAAUUAGUTT-3'; NC, 5'-UUCUCCGA ACGUGUCACGUTT-3'. The plasmid of pcDNA3.0-Trx1Flag was purchased from Addgen (Teddington, UK). The transfection of siRNA and plasmid was performed using Lipofectamine ${ }^{\circledR} 2000$ (Invitrogen, Carlsbad, CA, USA) following the manufacturer's protocol.

\section{Cell proliferation assays}

FLSs were plated at a density of $2 \times 10^{3}$ cells/well in 96-well plates and cultured for different time periods. At the end of each time period, cell proliferation was determined by using the CellTiter 96® Aqueous One Solution Cell Proliferation Assay kit (Promega, USA) according to the manufacturer's instructions. Briefly, $20 \mu \mathrm{l}$ 3-(4,5-dimethylthiazol-2-yl)-5-(3carboxymethoxyphenyl)-2-(4-sulfophenyl)-2H-tetrazolium, inner salt (MTS) was added to each well containing $100 \mu \mathrm{l}$ medium and then incubated at $37^{\circ} \mathrm{C}$ for $4 \mathrm{~h}$. The absorbance was read at $490 \mathrm{~nm}$ on a spectrophotometric plate reader (BioRad, Hercules, CA, USA) with a reference wavelength at $650 \mathrm{~nm}$. Each assay was performed in quintuplicate, and all tests were repeated three times.

\section{Western blot analysis}

The RA-FLSs were lysed in lysis buffer and centrifuged at $14,000 \mathrm{rpm}$ for $15 \mathrm{~min}$. The protein samples in the supernatant were immediately collected and the concentration was measured using the Bradford method (Bio-Rad, Hercules, CA, USA). Equal amounts of protein were separated by 10 or $15 \%$ SDSPAGE and then transferred onto nitrocellulose membranes (Amersham Pharmacia Biotech, Uppsala, Sweden). After blocking with 5\% skimmed milk in PBS containing $0.1 \%$ Tween-20 for $2 \mathrm{~h}$ at room temperature, the membranes were incubated with primary antibodies against Trx1, PI3Kp85 (no. 4292S), Akt (no. 9272), p-Akt (Ser473) (no. 4060S), Bcl-2 (no. 2870S) and Bax (no.m5023S) (all purchased from Cell Signal Technology Inc., USA), Caspase3 and active Caspase3 (ab32351 and ab13847 purchased from Abcam, Cambridge, MA, USA), and $\beta$-actin (sc-47778) (Santa Cruz Biotechnology Inc., USA), and subsequently incubated with secondary horseradish peroxidase antibody. The immunoreactive proteins were visualized using an enhanced chemiluminescent reagent (Millipore Corporation, USA).

\section{Flow cytometry analysis of apoptosis}

The trypsinized cells were collected and cell apoptosis was determined using BD Pharmingen ${ }^{\mathrm{TM}}$ FITC Annexin V Apoptosis Detection Kit I (BD Biosciences, USA) according to the manufacturer's instructions. The cells were washed twice with cold PBS and then resuspended in $500 \mu 11 \times$ binding buffer to a concentration of $1 \times 10^{6}$ cells $/ \mathrm{mL}$. After adding $5 \mu \mathrm{FITC}$ Annexin $\mathrm{V}$ and $5 \mu$ propidium iodide (PI), the cells were gently vortex and incubated at room temperature in the dark for $15 \mathrm{~min}$ and finally analyzed by a flow cytometer (Beckman Coulter, Fullerton, CA, USA). 


\section{Statistical analysis}

The experiments were done in triplicate in six different donors. Data were expressed as mean \pm SD. Statistical analysis was done with the mean of the triplicates of each donor $(n=6)$. All statistical analysis was performed by using the statistical software SPSS 17.0 (SPSS, Inc., Chicago, USA). Paired samples were analyzed with $t$ test. Block design was performed with Friedman M test. Two comparisons of multiple correlated samples were analyzed by $q$ test. It was considered statistically significant when a $p$ value was less than 0.05 .

\section{Result}

\section{Trx1 is abundant in RA synovial tissues}

To explore the distribution of Trx1 in RA and OA synovial tissues, we performed immunohistochemical staining. The results showed that Trx1 was expressed in both RA and OA synovial tissues. Trx1 was mainly localized in the cytosol and occasionally in the nucleus. Trx1 immunopositive cells were distributed diffusely (Fig. 1a). Furthermore, we detected the mRNA expression of Trx1 in synovial tissues by quantitative RT-PCR and found that Trx1 level was higher in RA synovial tissues than in OA (Fig. 1b). These results demonstrate that Trx1 is abundant in RA synovial tissues.

\section{Hypoxia promotes FLSs proliferation and induces Trx1 expression}

To investigate the effect of hypoxia on FLSs proliferation and Trx1 expression, RA-FLSs were cultured under conditions of different oxygen concentration $\left(3 \% \mathrm{O}_{2}\right.$ and $\left.21 \% \mathrm{O}_{2}\right)$ for different time periods. FLSs under hypoxia $\left(3 \% \mathrm{O}_{2}\right)$ condition grew faster than those under normoxic $\left(21 \% \mathrm{O}_{2}\right)$ (Fig. 2a). Subsequently, we analyzed the effect of hypoxia on the gene and protein expression of Trx1 in FLSs using quantitative RT-PCR (Fig. 2b) and Western blot (Fig. 2c), respectively. mRNA and protein levels of Trx 1 were consistently upregulated in the hyperactive FLSs cultured under $3 \% \mathrm{O}_{2}$ condition in a time-dependent manner as compared with those in FLSs under $21 \% \mathrm{O}_{2}$.

\section{Trx1 affects the proliferation of RA-FLSs}

To evaluate the effect of Trx1 on the proliferation of RAFLSs, we modulated Trx1 expression levels in FLSs by transfection with Trx1-siRNAs (si-Trx1-1 and si-Trx1-2) or a Trx1 expression construct. The transfected cells were then cultured under hypoxia or normoxic condition. Trx1 knockdown effectively attenuated hypoxia-induced proliferation of FLSs at 48, 72 , and $96 \mathrm{~h}$ after treatment as determined by MTS assay (Fig. 3a). Under normoxic condition, Trx 1 knockdown showed no effects at other time points (Fig. 3b). On the contrary, Trx 1 overexpression increased the proliferation of FLSs under normoxic condition (Fig. 3c) but had no effects on under hypoxia condition (Fig. 3d).

\section{Trx1 affects the apoptosis of RA-FLSs}

We then evaluated the effect of Trx1 on the apoptosis of RAFLSs. Following transfection of Trx1-siRNAs, the percentages of early apoptotic FLSs were significantly increased under hypoxia condition and were not changed under normoxic condition (Fig. 4a, b). To further confirm the role of Trx1 in the apoptosis of FLSs, we used Adriamycin (ADR), a well-known apoptosis-inducing agent, to treat FLSs. Flow cytometry analysis showed that Trx1 overexpression
A

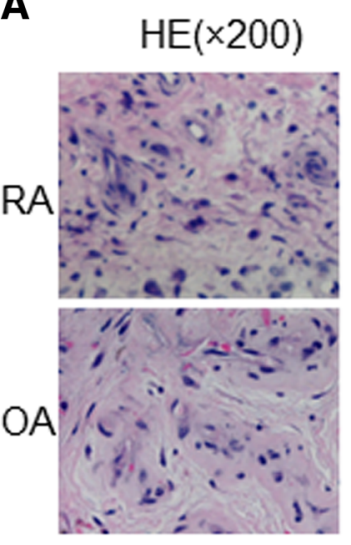

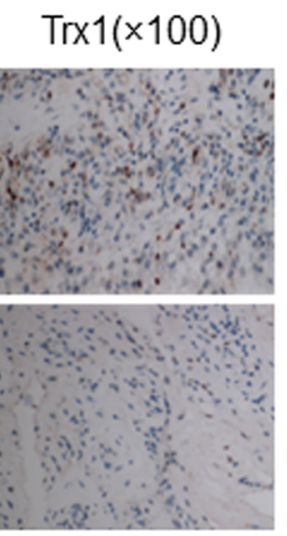

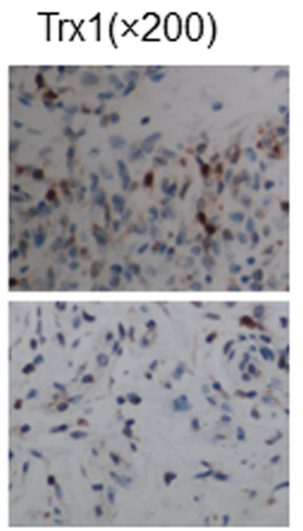

B

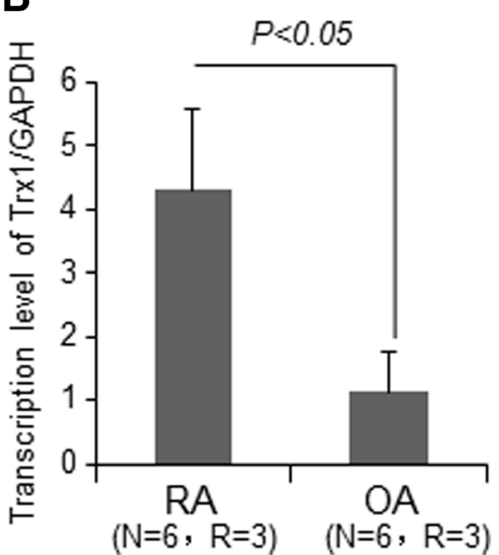

Fig. 1 Trx 1 is highly expressed in RA synovial tissues. a Immunohistochemical staining of Trx 1 in synovium from RA and OA patients. b The mRNA levels of Trx1 in synovial tissues by quantitative RT-PCR. The data in $\mathbf{b}$ are means \pm SD obtained from three separate experiments 

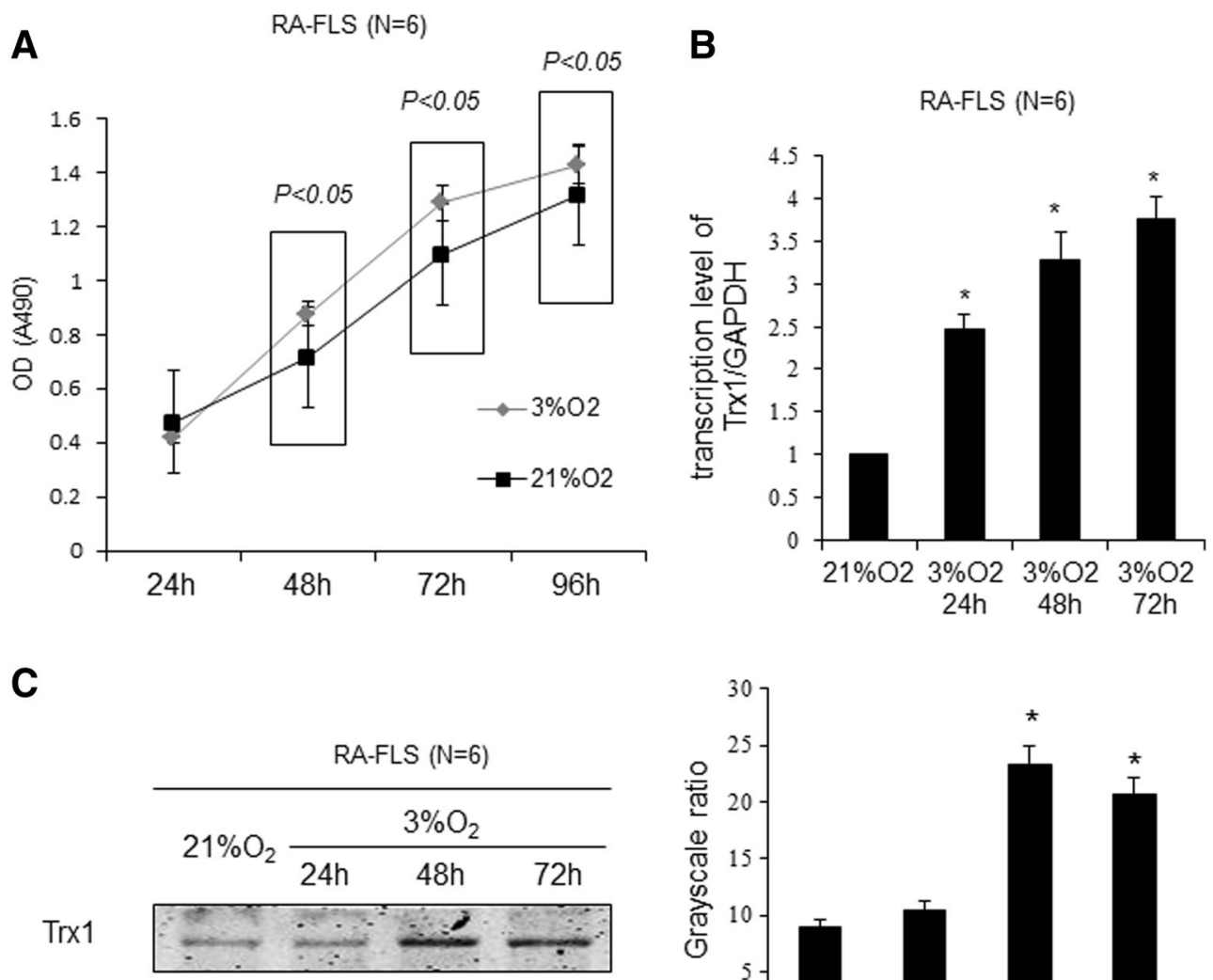

$\beta$-actin
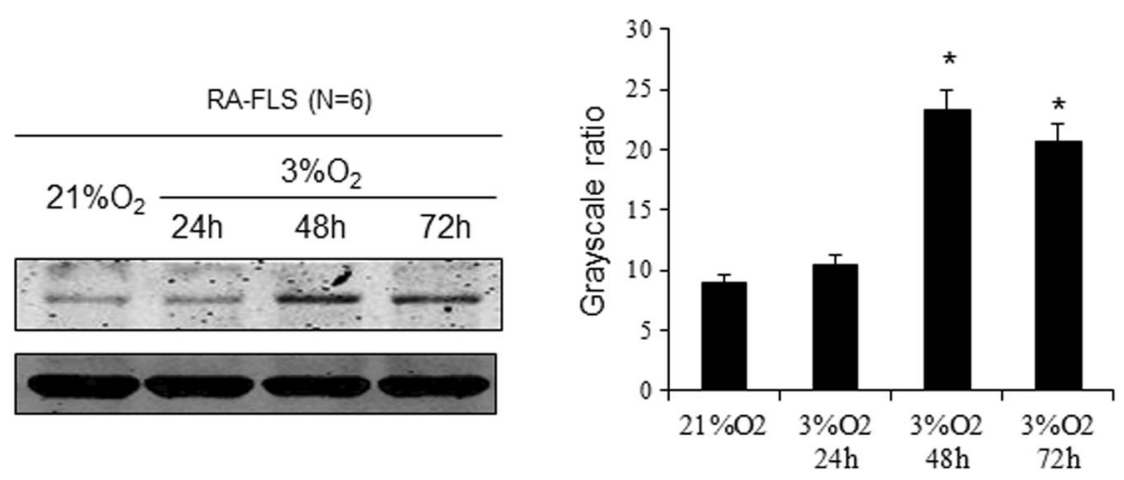

Fig. 2 Hypoxia promotes RA-FLS proliferation and induces Trx1 expression in FLSs. a Effect of different oxygen concentrations on cell proliferation as assessed by MTS assay. b Trx 1 mRNA expression levels were measured by quantitative RT-PCR analysis

diminished ADR-induced apoptosis of FLSs under both normoxic and hypoxia condition (Fig. 4c, d).

\section{Trx1 modulates PI3K-Akt activation and the expression apoptosis-related proteins in RA-FLSs}

To elucidate the molecular mechanism underlying Trx 1attenuated apoptosis of RA-FLSs, the activation of PI3KAkt and the expression of apoptosis-related proteins in RAFLSs following incubation in hypoxia were detected. Trx 1 knockdown significantly attenuated the protein level of PI3Kp85 and the phosphorylation of Akt at Ser473 in response to hypoxic exposure (Fig. 5A). Meanwhile, the apoptosis-related protein, Bcl-2, was also downregulated, but the active Caspase 3 and Bax were upregulated by Trx 1 knockdown in hypoxic FLSs (Fig. 5a).

To further confirm the role of $\operatorname{Trx} 1$ on the activation of PI3K-Akt, we overexpressed $\operatorname{Trx} 1$ in RA-FLSs under normoxic condition and assessed the levels of PI3Kp85, phospho-Akt, and apoptosis-related proteins. As shown in in RA-FLSs. c Trx1 protein expression levels were evaluated by Western blot analysis in RA-FLSs. The data are showed as means $\pm \mathrm{SD} .(* P<0.05$ compared with the group that was cultured under $21 \% \mathrm{O}_{2}$ )

Fig. 5b, Trx 1 overexpression evidently increased the levels of PI3Kp85, phospho-Akt, and Bcl-2 and decreased the levels of active Caspase 3 and Bax under normoxic condition. In addition, the pharmacological PI3K inhibitor LY-294002 significantly repressed the effects of $\operatorname{Trx} 1$ overexpression on the expression of the above proteins.

\section{Discussion}

FLSs from RA patients were found to display uncontrolled proliferation, which is causally related to the hypoxic microenvironment [27]. Our previous study has found that hypoxic exposure promotes the proliferation and reduces the apoptosis of RA-FLSs [28]. The increased level of $\operatorname{Trx} 1$ is closely correlated with the disease activity of RA, suggesting that Trx 1 may become a useful clinical biomarker of RA [21-23]. However, little is known about the effect of $\operatorname{Trx} 1$ on the biological behavior of RA-FLSs. 

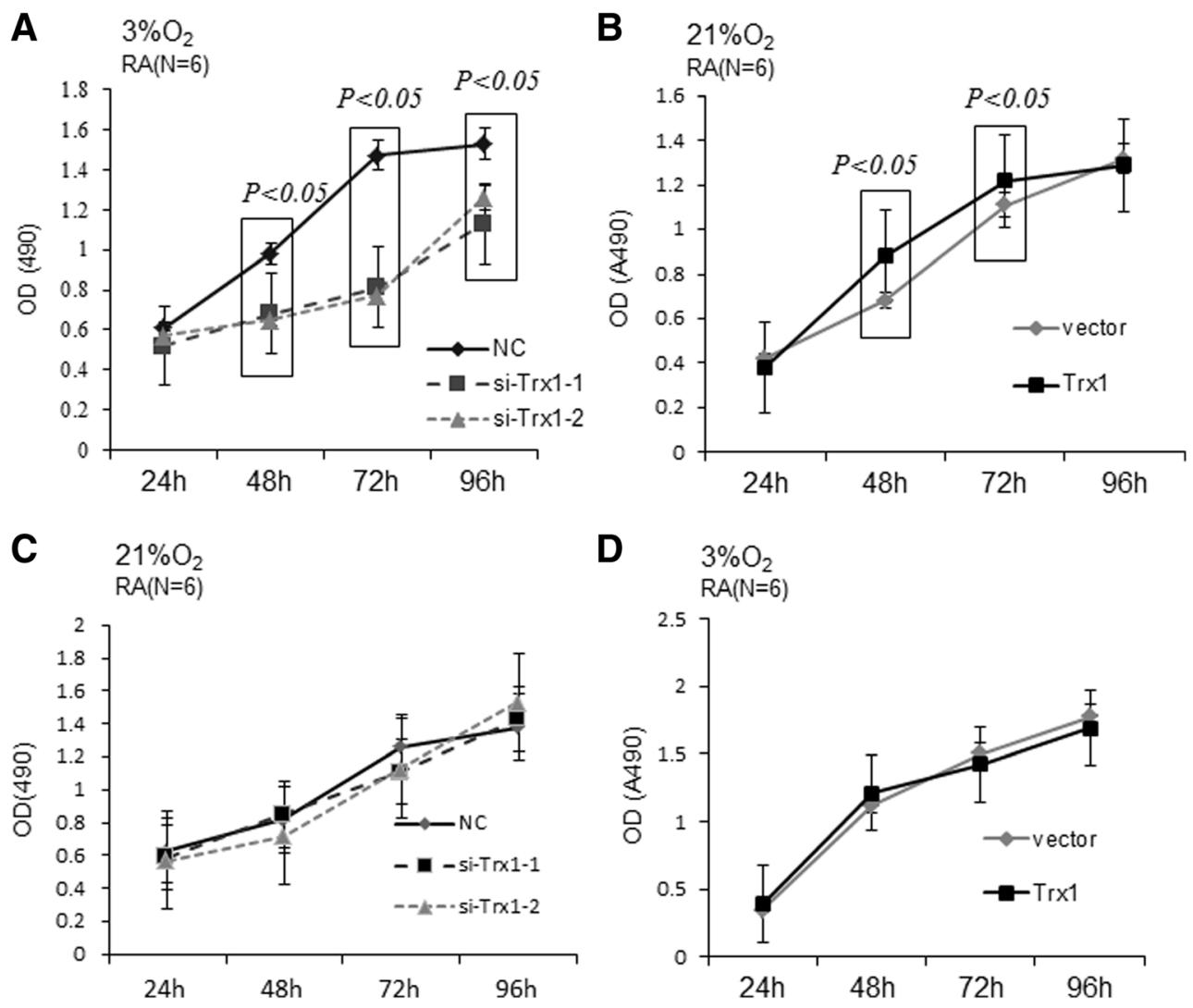

Fig. 3 Trx 1 affects the proliferation of RA-FLSs. a, b RA-FLSs were transfected with Trx1-siRNAs (si-Trx1-1 and si-Trx1-2) or control siRNA (NC) and cultured under $3 \% \mathrm{O}_{2}(\mathbf{a})$ and $21 \% \mathrm{O}_{2}$ (b) condition. The growth curve of RA-FLSs under $3 \% \mathrm{O}_{2}$ condition was determined by

MTS assay. c, d RA-FLSs were transfected with a Trx 1 expression construct or control vector and cultured under $21 \% \mathrm{O}_{2}$ condition. The growth curve of RA-FLS under $21 \% \mathrm{O}_{2}$ (c) and $3 \% \mathrm{O}_{2}$ (d) condition was assessed by MTS assay. The data are shown as means \pm SD

Trx1 has been shown to regulate the proliferation and survival of tumor cells and prevent oxidative stress-induced apoptosis of tumor cells [29]. In our previous study, we found that the expression of Trx1 was higher in RA-FLSs than that in OA-FLSs [30]. In the present study, we found that the Trx1 levels were significantly increased in synovial tissues from RA patients compared with those in OA. In addition, we found that hypoxic condition significantly induced the proliferation of RA-FLSs and the Trx 1 expression. These data suggest that hypoxia may be an inducer of Trx1 expression in RA-FLSs, which is intimately linked with the excessive proliferation.

One of the important pathophysiological characteristics of RA is synovial hyperplasia which leads to the destruction of articular cartilage and bone. The synovial hyperplasia is mainly due to an overabundance of FLSs. The overgrowth of FLSs in RA is largely from an imbalance between cell proliferation, survival, and death $[1,2]$. In this study, we found that Trx 1 knockdown effectively caused a significant decline in RAFLS proliferation accompanied with increased cell apoptosis under hypoxia condition. However, Trx 1 overexpression did not affect FLSs proliferation under hypoxia condition. It may be explained by that the increased Trx 1 by hypoxia was

sufficient to promote FLSs proliferation, and the ectopic overexpression of Trx1 had no addition effect. Meanwhile, Trx 1 knockdown showed limited inhibitory effect on FLSs proliferation, but Trx1 overexpression significantly promoted the proliferation of RA-FLSs under normoxic condition. The possible cause of these findings was that RA-FLSs under normoxic condition had relative low expression of Trx 1 and Trx1 knockdown could not further inhibit the proliferation of such cells. These results strongly imply that $\operatorname{Trx} 1$ is a key biological factor for the hyperproliferation and survival of RA-FLSs under hypoxia condition.

The PI3K-Akt pathway is abnormally activated in RAFLSs, which inhibits cell apoptosis and promotes cell proliferation by affecting multiple downstream effector molecules [31]. Apoptosis is subtly regulated by anti- and proapoptotic molecules. Caspase 3 is one of the most important proteases and plays a key role in the execution of apoptosis with other caspase cascades. Pro-apoptotic Bax and antiapoptotic Bcl-2 are the most important members of Bcl-2 family which affect cell growth by modulating cell apoptosis. In this study, we found that knockdown of Trx1 expression inhibited the PI3K expression and Akt activation in RA-FLSs following incubation in hypoxia. Furthermore, 
A

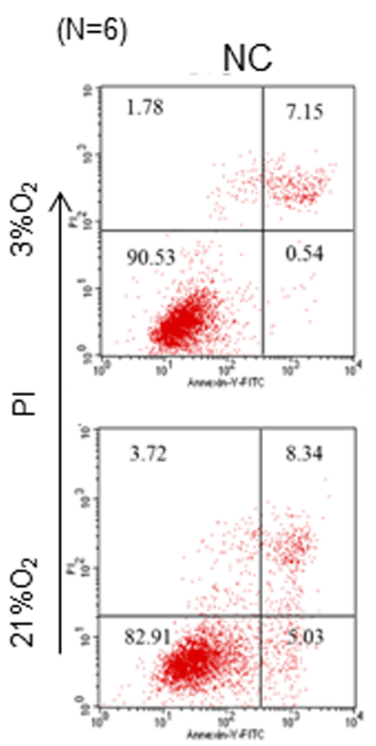

C
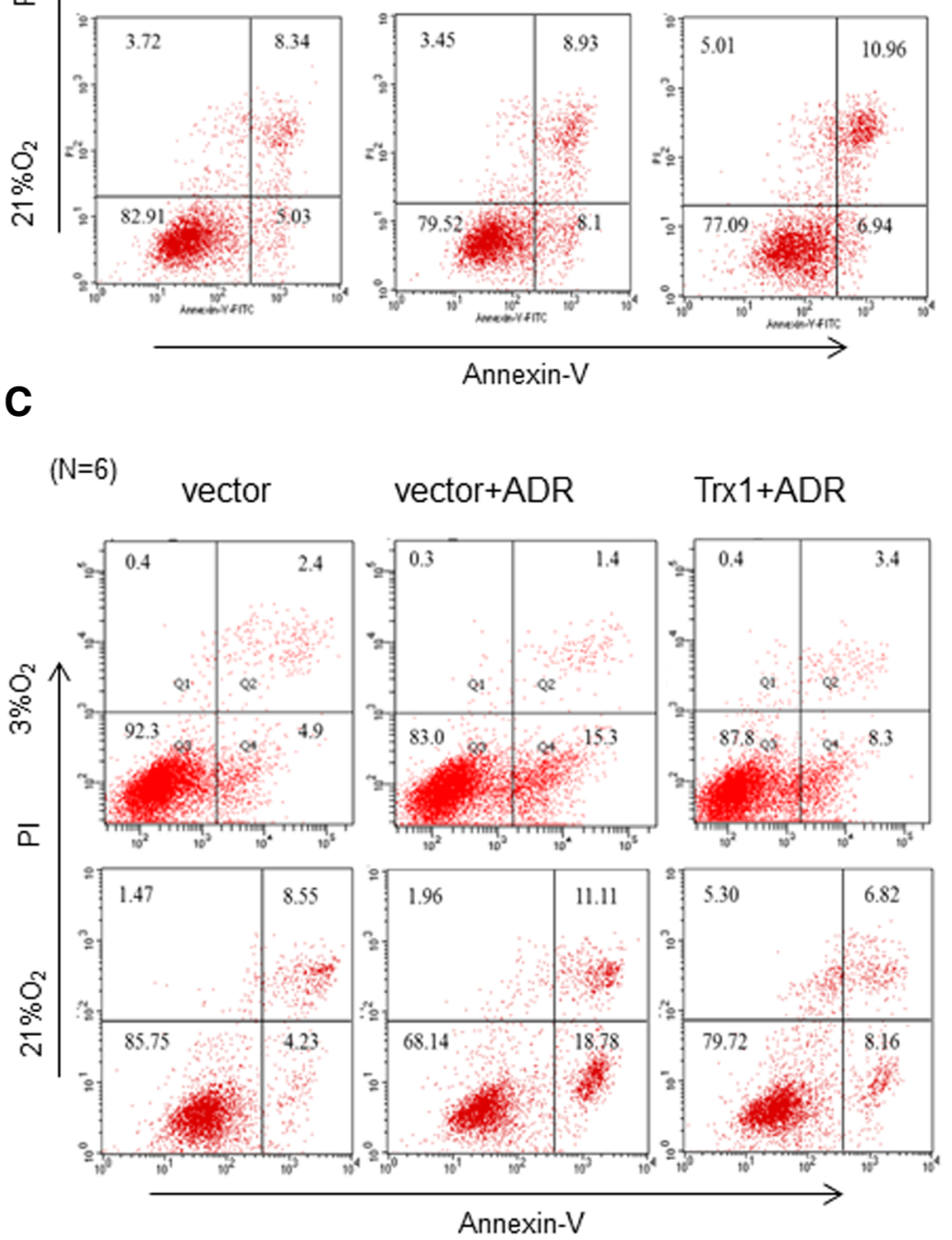

Annexin- $V$

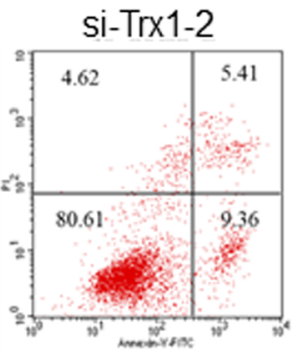

B
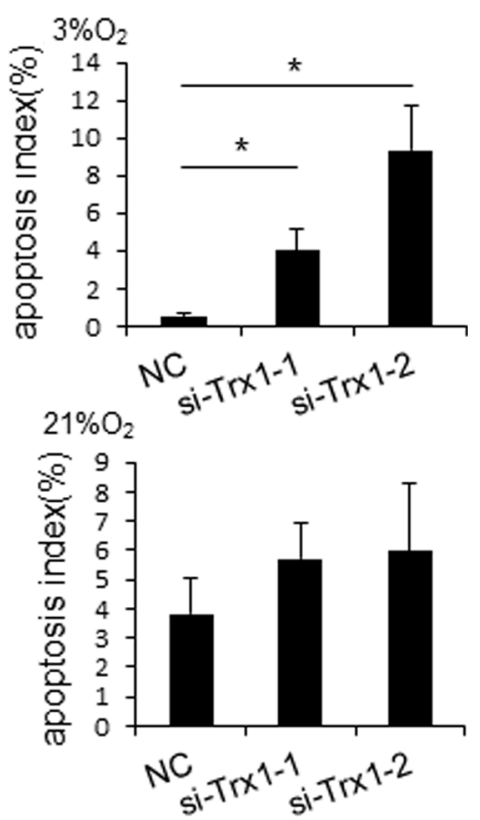

D

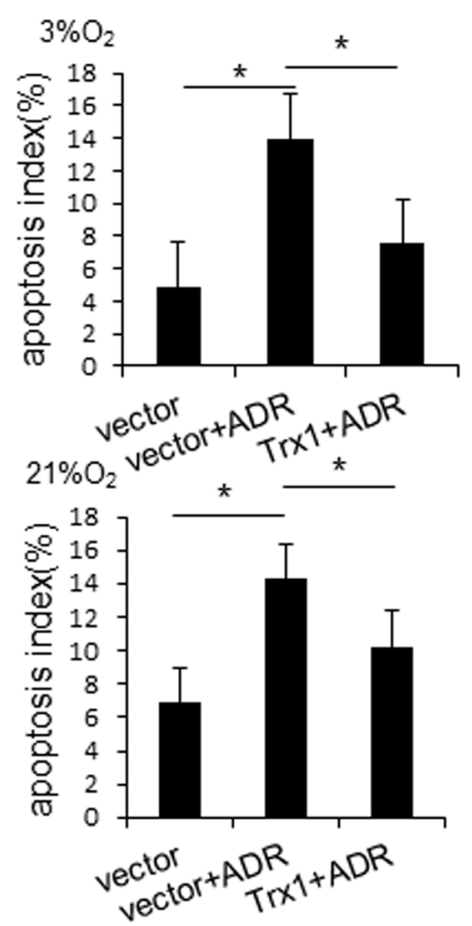

Fig. 4 Trx1 affects the apoptosis of RA-FLSs. a RA-FLSs were transfected with Trx1-siRNAs (si-Trx1-1 and si-Trx1-2) or control siRNA (NC) and cultured for $48 \mathrm{~h}$ under $3 \% \mathrm{O}_{2}$ and $21 \% \mathrm{O}_{2}$ conditions. Cell apoptosis was then detected by flow cytometry. b Bar graph data of the early apoptotic cells (lower-right quadrant) are shown. c RA-FLSs were transfected with a Trx1 expression construct or control vector. After 48 -h culture under $3 \% \mathrm{O}_{2}$ and $21 \% \mathrm{O}_{2}$

we found that Trx 1 knockdown caused a significant reduction in the expression of Bcl-2 but enhanced the expression of active Caspase 3 and Bax in hypoxic RA-FLSs. These conditions, FLSs were incubated in the presence of $1 \mu \mathrm{g} / \mathrm{mL}$ of Doxorubicin (Adriamycin, ADR; Sangon, Shanghai, China) for $24 \mathrm{~h}$. Cell apoptosis was then detected by flow cytometry. d Bar graph data of the early apoptotic cells (lower-right quadrant) are shown. The data in $\mathbf{a}$ and $\mathbf{c}$ are representatives of three separate experiments, and in $\mathbf{b}$ and $\mathbf{d}$ are shown as mean $\pm \mathrm{SD}(* P<0.05$, $* * P<0.01)$

findings suggest that Trx1 inhibits RA-FLSs apoptosis by regulating activation of PI3K-Akt under hypoxic conditions. In normoxia, Trx1 overexpression evidently 
Fig. 5 Trx1 modulates PI3K-Akt activation and the expression apoptosis-related proteins in RAFLSs. a, c RA-FLSs were transfected with Trx1-siRNAs (siTrx1-1 and si-Trx 1-2) or control siRNA (NC) and cultured for $48 \mathrm{~h}$ under $3 \% \mathrm{O}_{2}$ condition. Western blot and grayscale ratio analyses for the activation of PI3K-Akt and the expression of apoptosisrelated proteins in RA-FLSs are shown. $* P<0.05$. b, d RA-FLSs were transfected with a Trx1 expression construct or control vector. After 48-h culture under $21 \% \mathrm{O}_{2}$ condition, $20 \mu \mathrm{M} \mathrm{LY}-$ 294002 (Beyotime

Biotechnology, Jiangsu, China) or an equal volume of DMSO was added to the cultured media. Cells were then incubated under normoxia for $24 \mathrm{~h}$ and Western blot analyses were then performed. Representative blot and grayscale ratio analyses of three separate experiments are shown $\# P<0.05$ versus vectortransfected cells, $\_$\& $P<0.05$ Trx 1-transfected cells versus Trx1-transfected and LY-294002treated cell)
A
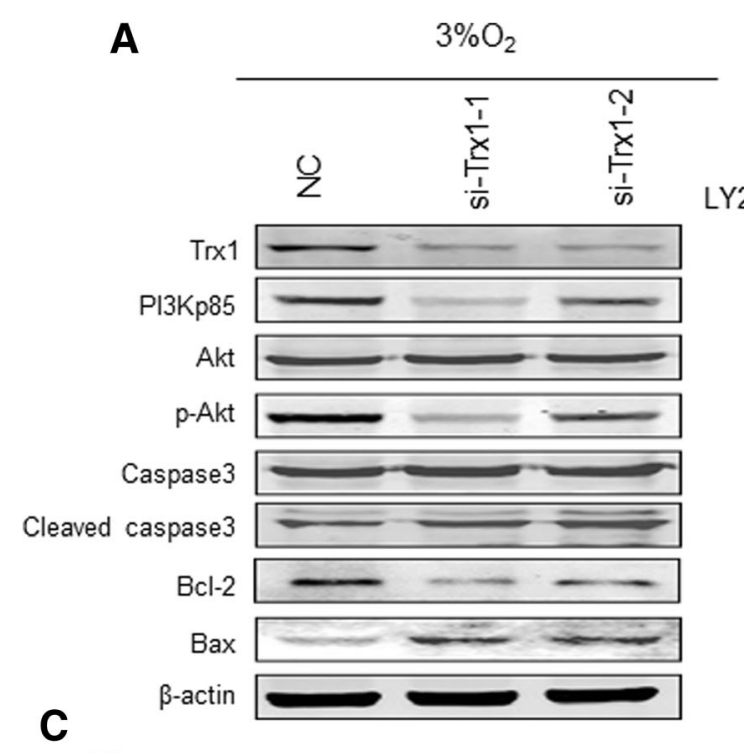

B $21 \% \mathrm{O}_{2}$

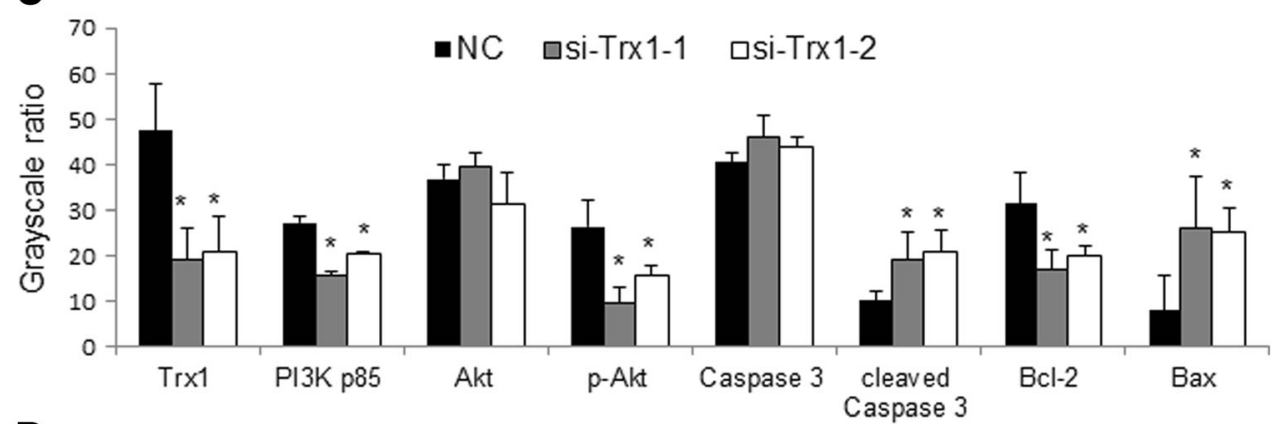

D

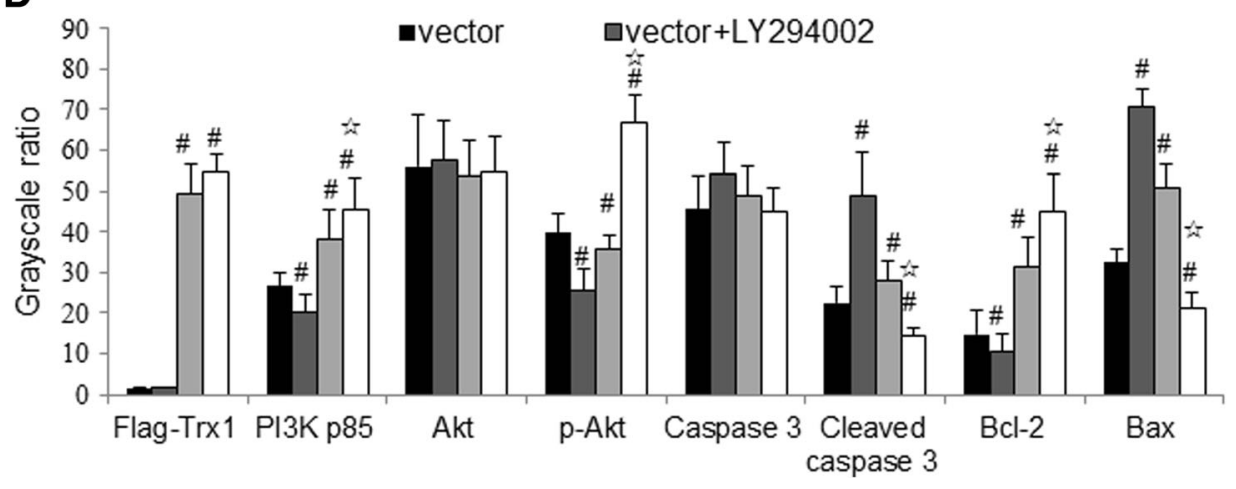

promoted the expression of PI3K and phospho-Akt in RAFLSs. Moreover, we found that the upregulation of Trx1 markedly increased the expression of Bcl-2 and decreased the expressions of active Caspase 3 and Bax under normoxic conditions. Such effects were partially suppressed by LY-294002, an inhibitor of PI3K. Our results also provide evidence that Trx 1 and PI3K-Akt pathway may exert anti-apoptotic role under normoxic conditions.

In conclusion, our data strongly suggest that Trx1 may play a crucial pathophysiologic role in RA through the promotion of hyperproliferation of FLSs via a signaling pathway which depends on the activation of PI3K-Akt. Moreover, we have identified and demonstrated that Trx1 is highly expressed in
RA-FLSs cultured under hypoxic conditions. Therefore, Trx1 may be a promising therapeutic target for RA.

Acknowledgements We thank the patients for their contribution to this study and all the members of the lab for stimulating discussion. This project was supported by the Natural Science Foundation of China (81373203, 81601407, and 81671599), Shanghai Municipal Health and Planning Commission (no. 20154Y0115), and Pudong New Area Science and Technology Commission (PKJ2015-Y18, PW2013B-5).

\section{Compliance with ethical standards}

Disclosures None. 
Open Access This article is distributed under the terms of the Creative Commons Attribution 4.0 International License (http:// creativecommons.org/licenses/by/4.0/), which permits unrestricted use, distribution, and reproduction in any medium, provided you give appropriate credit to the original author(s) and the source, provide a link to the Creative Commons license, and indicate if changes were made.

\section{References}

1. Bottini N, Firestein GS (2012) Duality of fibroblast-like synoviocytes in RA: passive responders and imprinted aggressors. Nat Rev Rheumatol 9(1):24-33

2. Bartok B, Firestein GS (2010) Fibroblast-like synoviocytes: key effector cells in rheumatoid arthritis. Immunol Rev 233(1):233-255

3. Ganesan R, Rasool M (2017) Fibroblast-like synoviocytes-dependent effector molecules as a critical mediator for rheumatoid arthritis: current status and future directions. Int Rev Immunol 36(1):20-30

4. Muz B, Khan MN, Kiriakidis S, Paleolog EM (2009) Hypoxia. The role of hypoxia and HIF-dependent signalling events in rheumatoid arthritis. Arthritis Res Ther 11(1):201

5. Juranek I, Stern R, Soltes L (2014) Hyaluronan peroxidation is required for normal synovial function: an hypothesis. Med Hypotheses 82(6):662-666

6. Sivakumar B, Akhavani MA, Winlove CP, Taylor PC, Paleolog EM, Kang N (2008) Synovial hypoxia as a cause of tendon rupture in rheumatoid arthritis. J Hand Surg 33(1):49-58

7. Ng C, Biniecka M, Kennedy A, McCormick J, Fitzgerald O, Bresnihan B et al (2010) Synovial tissue hypoxia and inflammation in vivo. Ann Rheum Dis 69(7):1389-1395

8. Kennedy A, Ng CT, Biniecka M, Saber T, Taylor C, O'sullivan J et al (2010) Angiogenesis and blood vessel stability in inflammatory arthritis. Arthritis Rheum 62(3):711-721

9. Biniecka M, Kennedy A, Fearon U, Ng CT, Veale DJ, O'Sullivan JN (2009) Oxidative damage in synovial tissue is associated with in vivo hypoxic status in the arthritic joint. Ann Rheum Dis 69(6): $1172-1178$

10. Huber L, Distler O, Tarner I, Gay R, Gay S, Pap T (2006) Synovial fibroblasts: key players in rheumatoid arthritis. Rheumatology 45(6):669-675

11. Yan W, Fu Y, Tian D, Liao J, Liu M, Wang B et al (2009) PI3 kinase/Akt signaling mediates epithelial-mesenchymal transition in hypoxic hepatocellular carcinoma cells. Biochem Biophys Res Commun 382(3):631-636

12. Li GQ, Zhang Y, Liu D, Qian Y-Y, Zhang H, Guo SY et al (2013) PI3 kinase/Akt/HIF-1 $\alpha$ pathway is associated with hypoxiainduced epithelial-mesenchymal transition in fibroblast-like synoviocytes of rheumatoid arthritis. Mol Cell Biochem 372(1-2): 221-231

13. Carnero A (2010) The PKB/AKT pathway in cancer. Curr Pharm Des 16(1):34-44

14. Zhang Y, Zhang B, Trichostatin A (2015) An inhibitor of histone deacetylase, inhibits the viability and invasiveness of hypoxic rheumatoid arthritis fibroblast-like synoviocytes via PI3K/Akt signaling. J Biochem Mol Toxicol 30(4):163-169

15. Collet JF, Messens J (2010) Structure, function, and mechanism of thioredoxin proteins. Antioxid Redox Signal 13(8):1205-1216
16. Welsh SJ, Bellamy WT, Briehl MM, Powis G (2002) The redox protein thioredoxin-1 (Trx-1) increases hypoxia-inducible factor $1 \alpha$ protein expression. Cancer Res 62(17):5089-5095

17. Zhou J, Damdimopoulos AE, Spyrou G, Brüne B (2007) Thioredoxin 1 and thioredoxin 2 have opposed regulatory functions on hypoxia-inducible factor- $1 \alpha$. J Biol Chem 282(10): $7482-7490$

18. Sartelet H, Rougemont A-L, Fabre M, Castaing M, Duval M, Fetni $\mathrm{R}$ et al (2011) Activation of the phosphatidylinositol 3'-kinase/AKT pathway in neuroblastoma and its regulation by thioredoxin 1 . Human Pathology 42(11):1727-1739

19. Meuillet EJ, Mahadevan D, Berggren M, Coon A, Powis G (2004) Thioredoxin-1 binds to the C2 domain of PTEN inhibiting PTEN's lipid phosphatase activity and membrane binding: a mechanism for the functional loss of PTEN's tumor suppressor activity. Arch Biochem Biophys 429(2):123-133

20. Chen B, Nelin VE, Locy ML, Jin Y, Tipple TE (2013) Thioredoxin1 mediates hypoxia-induced pulmonary artery smooth muscle cell proliferation. Am J Phys Lung Cell Mol Phys 305(5):389-395

21. Jikimoto T, Nishikubo Y, Koshiba M, Kanagawa S, Morinobu S, Morinobu A et al (2002) Thioredoxin as a biomarker for oxidative stress in patients with rheumatoid arthritis. Mol Immunol 38(10): $765-772$

22. Heim K, Dälken B, Faust S, Rharbaoui F, Engling A, Wallmeier H et al (2016) High thioredoxin-1 levels in rheumatoid arthritis patients diminish binding and signalling of the monoclonal antibody Tregalizumab. Clin Transl Immunol 5(12):e121

23. Hawkes HJ, Karlenius TC, Tonissen KF (2014) Regulation of the human thioredoxin gene promoter and its key substrates: a study of functional and putative regulatory elements. Biochim Biophys Acta 1840(1):303-314

24. Burke-Gaffney A, Callister ME, Nakamura H (2005) Thioredoxin: friend or foe in human disease? Trends Pharmacol Sci 26(8):398404

25. Aletaha D, Neogi T, Silman AJ, Funovits J, Felson DT, Bingham $\mathrm{CO}$ et al (2010) Rheumatoid arthritis classification criteria: an American College of Rheumatology/European League Against Rheumatism collaborative initiative. Arthritis Rheum 62(9):2569 2581

26. Madry H, Kon E, Condello V, Peretti GM, Steinwachs M, Seil R et al (eds) (2016) Early osteoarthritis of the knee. Knee Surg Sports Traumatol Arthrosc 24(6):1753-1762

27. Nonomura Y, Mizoguchi F, Suzuki A, Nanki T, Kato H, Miyasaka $\mathrm{N}$ et al (2009) Hypoxia-induced abrogation of contact-dependent inhibition of rheumatoid arthritis synovial fibroblast proliferation. J Rheumatol 36(4):698-705

28. Fan SS, Zong M, Zhang H, Lu Y, Lu TB, Fan LY (2015) Decreased expression of alpha-enolase inhibits the proliferation of hypoxiainduced rheumatoid arthritis fibroblasts-like synoviocytes. Mod Rheumatol 25(5):701-707

29. Kaimul AM, Nakamura H, Masutani H, Yodoi J (2007) Thioredoxin and thioredoxin-binding protein- 2 in cancer and metabolic syndrome. Free Radic Biol Med 43(6):861-868

30. Zhang H, Fan L, Zong M, Sun L, Lu L (2012) Proteins related to the functions of fibroblast-like synoviocytes identified by proteomic analysis. Clin Exp Rheumatol 30(2):213-221

31. Bartok B, Boyle DL, Liu Y, Ren P, Ball ST, Bugbee WD et al (2012) PI3 kinase $\delta$ is a key regulator of synoviocyte function in rheumatoid arthritis. Am J Pathol 180(5):1906-1916 